\title{
Evaluation de l'impact de l'atteinte des cibles de couverture des indicateurs des plans en vigueur sur la sous nutrition et la mortalité des enfants de moins de 5 ans au Mali
}

\section{Evaluation of the impact of reaching the targets for covering the indicators of the plans in force on undernutrition and mortality of children under 5 in Mali}

\author{
Touré $\mathrm{HM}^{1}$, Kéita $\mathrm{Y}^{2}, \mathrm{Ag}$ Iknane $\mathrm{A}^{1}$, Téréra $\mathrm{I}^{1}$
}

1/nstitut National de Santé Publique (INSP), Bamako, Mali

2Institute for International Programs, Johns Hopkins University

Bloomberg School of Public Health, Bamako, Mali

\begin{abstract}
Résumé
Introduction : Au Mali, le niveau de la sous nutrition des enfants de moins de 5 ans restent préoccupants. L'objectif de cette étude était d'évaluer l'impact de l'atteinte des cibles de couverture des indicateurs de plans SMNI\&N en vigueur au Mali sur la sous nutrition et la mortalité des enfants de moins de 5 ans. Matériel et méthodes : Nous avons modélisé l'impact de deux paquets d'intervention dans LiST entre 2014 et 2023. Une projection incluait les interventions et les cibles des plans en vigueur en 2014. Une autre projection portait à une couverture de $100 \%$ les interventions nutritionnelles et de WASH. Résultats : Nos résultats montrent que, si le Mali atteint les cibles des plans, plus de 115800 cas de malnutrition chronique seraient évités en 2018 et jusqu'à plus de 711000 cas en 2023. Les pratiques appropriées d'alimentation de complément éviteraient plus de 364000 cas. La malnutrition chronique serait réduite de $38 \%$ à $33,9 \%$ contre $31,9 \%$ pour le scenario. La malnutrition aigüe serait réduite de $12,9 \%$ à $9,9 \%$ contre $8,8 \%$ pour le scénario. Le nombre de vies sauvées chez les enfants serait de plus de 71500 en 2018 et plus de 214000 en 2023. La réduction la plus importante s'observerait pour la mortalité infanto-juvénile qui passerait à 126,6 à 80\% naissances vivantes en 2023 . Conclusion : L'étude a révélé que, même avec la mise à échelle des interventions à une couverture de $100 \%$ du secteur de la Santé et du WASH, les objectifs de sousnutrition des plans ne seront pas atteints. D'où la nécessité de mener des actions multisectorielles coordonnées.
\end{abstract}

Mots Clés : Malnutrition, mortalité, Plate-forme d'évaluation nationale (NEP), LiST, Mali

\section{Summary}

Introduction: In Mali, the level of undernutrition among children under 5 remains a concern. The objective of this study was to evaluate the impact of the achievement of coverage targets of current MNCH \& N plan indicators on under-nutrition and under-five mortality. Material and methods: We modeled the potential impact of two intervention packages in LiST between 2014 and 2023. A projection included the interventions and targets of the plans in effect in 2014. Another projection involved 100\% coverage of nutritional and WASH interventions. Results: Our results show that if Mali were to achieve the targets of the plans, more than 115,800 cases of chronic malnutrition would be avoided in 2018, and this number exceeds up to 711,000 in 2023. Appropriate complementary feeding practices would avoid more than 364,000 case. Stunting would be reduced from $38 \%$ to $33.9 \%$ against $31.9 \%$ for the scenario. Wasting would be reduced from $12.9 \%$ to $9.9 \%$ against $8.8 \%$ for the scenario. The number of lives saved among children would be more than 71,500 in 2018 and more than 214,000 in 2023 . The most significant reduction would be observed for less than five mortality from 126.6 to 80 per 1000 births alive in 2023. Conclusion: Our study demonstrated that even with scale-up of interventions to up $100 \%$ coverage of health sector and WASH interventions, the under-nutrition goals of the plans will not be achieved. Then, there is need of coordinated multisectoral actions.

Keywords: Malnutrition, mortality, National Evaluation Platform (NEP), LiST, Mali

\section{Introduction}

La prévalence de la malnutrition maternelle et infantile est forte dans les pays à revenu faible et intermédiaire, ce qui entraîne une hausse substantielle de la mortalité et du poids global de la morbidité. Au niveau mondial, 165 millions d'enfants de moins de 5 ans souffrent de retard de croissance, 52 millions d'émaciation, et plus de 2 milliards de personnes souffrent de carence en micronutriment, principalement en vitamine $A$, Fer, lode et Zinc. L'Afrique subsaharienne et l'Asie du Sud abritent les trois quarts des enfants souffrant d'un retard de croissance [1].

La sous-nutrition (retard de croissance intra-utérin, allaitement non-optimal, maigreur, retard de croissance et les carences en vitamine $A$ et en zinc) est à l'origine de $45 \%$ des décès chez les enfants de moins de 5 ans [2].

Les mortalités maternelle et infantile demeurent un problème majeur de santé publique dans le monde et particulièrement en Afrique subsaharienne, qui abrite les six pays avec un taux de mortalité des moins de cinq ans supérieur à 100 décès pour 1000 naissances vivantes (NV). En 2016, 5,6 millions d'enfants sont morts avant leur cinquième anniversaire, parmi eux 2,6 millions (46\%) 
sont morts dans le premier mois de la vie [3]. De plus 300000 décès maternels ont lieu chaque année [4].

Au Mali, l'état nutritionnel des enfants de moins de 5 ans n'a pas connu d'amélioration entre 2001 et 2012. A l'exception de l'insuffisance pondérale qui a connu une légère baisse (de $33 \%$ à $26,4 \%$ ), les deux autres indicateurs ont eu une tendance stable voir même à la hausse. La prévalence du retard de croissance est passée de $38 \%$ à $38,3 \%$ et celle de l'émaciation est passée de $11 \%$ à $12,7 \%[5,6]$.

Concernant la mortalité maternelle et infantile, les progrès sont lents. Les taux de mortalité néonatale, infantile et infanto juvénile ainsi que le ratio de mortalité maternelle restent élevés. Entre 2001 et 2012, le taux de mortalité néonatale est passé de 54,7 à 38,8 pour 1000 NV, le taux de mortalité infantile de 111,9 à 75,1 pour $1000 \mathrm{NV}$, le taux de mortalité infanto-juvénile de 211,4 à 126,6 pour $1000 \mathrm{NV}$ [7], et le ratio de mortalité maternelle de 582 à 368 pour 100000 NV [5,6].

Cette situation est préoccupante pour les décideurs compte tenu du fait que le Mali a adhéré au concept de la redevabilité, conformément à la déclaration de Paris, pour les objectifs de la santé et doit honorer ses engagements avec la communauté internationale. Cela se concrétise par un suivi des indicateurs afin de renforcer la redevabilité et accélérer les progrès mondiaux en matière de nutrition et de développement durable.

La volonté du gouvernement à améliorer la santé maternelle et infantile s'est matérialisée à travers le Programme Décennal de Développement Sanitaire et Social (PDDSS) 2014-2023 qui est mis en œuvre à travers sa première tranche quinquennale (PRODESS III, 2014-2018) à partir duquel découlent les autres plans (nutrition, paludisme, santé de la reproduction, etc.).

Alors que le Mali n'a pas pu atteindre les Objectifs du Millénaire pour le Développement (OMD) 4 et 5 relatifs aux mortalités infantile et maternelle en 2015, les perspectives liées à la réalisation universelle des cibles $(3.1,3.2$.$) du troisième Objectif du Développement$ Durable (ODD) en 2030 en matière de santé maternelle et infantile sont peu probables si les tendances actuelles sont maintenues.

$\mathrm{Ce}$ constat suscite des questions de plus en plus complexes auxquelles les décideurs veulent avoir des réponses basées sur des évidences qui ne sont pas toujours disponibles à travers les méthodes d'évaluation traditionnelles. Ce qui permet d'identifier et prioriser les interventions prometteuses afin d'accélérer les progrès dans ce domaine.

C'est dans ce cadre que le Mali a mis en œuvre la Plateforme Nationale d'Evaluation (NEP) pour répondre aux exigences des rapports et comptes rendus de la communauté. La NEP est une nouvelle approche d'évaluation qui utilise les données disponibles au niveau des pays et prend en compte un ensemble de facteurs contextuels pour une meilleure évaluation des programmes. Elle permet aux gouvernements de suivre et d'évaluer la mise en œuvre simultanée de divers programmes de SMNI\&N. La NEP est mise en œuvre par l'Institute for international programs de l'Université Johns Hopkins (IIP-JHU) et financée pour la période 2014 2017 par Affaires Mondiales Canada (AMC).

Au cours du Cycle 1 de la NEP au Mali, il a été démontré que l'atteinte des cibles des plans en matière de réduction de la malnutrition chronique $(15 \%)$ et de la malnutrition aigüe (5\%) permettrait de sauver 127791 vies d'enfants de moins de 5 ans soit $47 \%$ du nombre total [8]. Toutefois la question fondamentale reste à savoir si les interventions prévues dans les plans permettront d'atteindre ces cibles de malnutrition en 2023. Ainsi, étant donné que la revue de la nutrition dans LiST fait partir des nouvelles directives de la NEP, il est important pour nous de saisir cette opportunité pour répondre à cette sous question du cycle 1 qui est encore prioritaire pour les autorités du Mali. La présente étude s'inscrit dans ce cadre et avait pour objectif d'évaluer l'impact de l'atteinte des cibles de couverture des indicateurs des plans en vigueur sur la sous nutrition et la mortalité des enfants de moins de 5 ans au Mali.

\section{Matériel et Méthodes \\ Schéma de l'étude}

Nous avons utilisé l'Outil de Vies Sauvées (Lives Saved Tool-LiST) pour mener une modélisation prospective des effets de deux paquets d'interventions sur la sous nutrition et la mortalité des enfants de moins de 5 ans au Mali, de 2014 à 2023. L'étude s'est déroulée entre octobre 2017 et février 2018.

\section{Cadre de l'étude}

Cette étude portait sur le niveau national. Cependant, bien que les plans fixent des objectifs nationaux pour la sous nutrition et la mortalité des enfants de moins de 5 ans, les données de l'EDSM $V$ utilisées comme niveaux de base pour la plupart des indicateurs ne prenaient pas en compte les trois régions du Nord du Mali (Gao, Tombouctou et Kidal) en raison de problèmes de sécurité dans le Nord du pays, ces régions n'étaient pas incluses dans l'EDS 2012 qui était la source la plus récente des données de couverture. Les cinq régions incluses dans l'EDSM sont Kayes, Koulikoro, Sikasso, Ségou, Mopti et le district de Bamako [6] et sont également celles sur lesquelles portent nos analyses.

\section{Données utilisées}

Les données utilisées pour les modélisations avec LiST ont été extraites, (i) des plans et stratégies en vigueur sur la SMNI\&N au Mali (Plan Décennal de Développement Sanitaire et Social (PDDSS) 2014-2023, Programme de 
Développement Sanitaire et Social (PRODESS III) 20142018, Plan Stratégique de la Santé de la Reproduction (PSR) 2014-2018), Plan Stratégique de Lutte contre le Paludisme (PSLP) 2013-2017, Plan d'Action Multisectorielle de Nutrition (PAMN) 2014-2018. (ii), de I"EDSM V (rapport et base de données), (iii), du Recensement Général de la Population et de l'Habitat (RGPH) de 2009, (iv) des données du groupe interagence onusien pour l'estimation de la mortalité des enfants (IGME) et(v) des données de couverture de vaccination de L'OMS/UNICEF. La plupart des indicateurs étaient disponibles dans les rapports, d'autres par contre ont été recalculés à partir de la base d'EDSM V pour répondre à la définition de LiST en utilisant le logiciel Stata 13.0.

Quand les données de référence des plans étaient tirées des sources de routine, elles ont été remplacées par les données provenant de l'EDSM V. Les données de vaccination de l'OMS/UNICE jugées plus précises ont été utilisées comme référence. Nous avons utilisé les données d'IGME pour les données de référence de mortalité des enfants de moins de 5 ans en raison des problèmes de qualité évoqués dans le rapport d'EDSM V relatifs aux données de mortalités [6]. Les cibles des plans ont été retenues comme cibles pour la modélisation LiST. Si un même indicateur était pris en compte par plus d'un plan, la cible la plus ambitieuse a été retenue pour la modélisation afin de maximiser l'impact des plans.

Modélisation et visualisation dans LiST

Nous avons utilisé la version 5.621 de LiST, qui est un modèle mathématique qui rassemble les meilleures données disponibles sur la population, la cause du décès, l'intervention, l'efficacité et la couverture pour estimer l'impact du changement dans la couverture de l'intervention sur la malnutrition et la mortalité des enfants de moins de 5 ans et des mères[9].

Deux modèles (projections) ont été construits pour la modélisation dans LiST. La première projection nationale contenait les niveaux de base et les cibles des plans en 2018 et 2023 pour chaque indicateur retenu. La seconde projection nationale est un scenario dans lequel en 2023, toutes les interventions (excepté la prévalence contraceptive, l'accouchement par du personnel qualifié et les SONUB) sont portées à l'échelle de $100 \%$ de couverture avec comme objectif d'estimer l'impact maximal des interventions du secteur de la santé et du WASH sur les indicateurs de la sous nutrition (malnutrition chronique et aigüe) (Tableau I).

Pour chaque modèle, l'année 2014 a été utilisée comme année de base et les années 2018 et 2023 comme années cibles car les plans quinquennaux pour la plupart s'arrêtaient en 2018 et le PDDSS en 2023. Si un indicateur était pris en compte dans le PDDSS et dans un plan quinquennal, nous avons procédé à une interpolation linéaire de 2014 à 2018 puis de 2018 à 2023. Pour les indicateurs qui n'avaient pas de cible en 2018 nous avons interpolé entre 2014 et 2023.

\section{Résultats}

Impact des interventions sur la sous nutrition des enfants de moins de 5 ans selon les plans SMNI\&N en vigueur au Mali de 2014 à 2023 Nombre de cas de malnutrition chronique évités chez les enfants de moins de 5 ans et par intervention

Si le Mali atteignait les cibles de ses plans, le nombre de cas malnutrition chronique évités chez les enfants de moins de 5 ans serait de 115866 en 2018 et jusqu'à 711202 en 2023 dont $45 \%$ chez les enfants de moins de 2 ans (Tableau II).

Les interventions qui éviteraient le plus de cas de malnutrition chronique étaient les pratiques appropriées d'alimentation de complément chez les nourrissons et jeunes enfants avec 364264 cas soit environ $52 \%$ du nombre total, suivis du lavage des mains au savon (109 685 cas) soit $15,5 \%$, de la supplémentation en vitamine A (51 835 cas) soit 7,3\% et de la connexion d'eau dans la maison (47 268 cas) soit 6,7\% (Graphique 1).

Les interventions nutritionnelles directes (ANJE, supplémentation en micronutriments) avec $65 \%$ et WASH $(31 \%)$ seraient responsables de $96 \%$ des cas de malnutrition chronique évités chez les enfants de moins de 5 ans (Graphique 2).

Réduction des prévalences de la malnutrition chronique et de la malnutrition aigüe chez les enfants de moins de 5 ans

La projection basée sur l'atteinte des cibles des plans réduirait la malnutrition chronique de $38 \%$ à $33,9 \%$ soit un taux de réduction de $11 \%$. Pour le scenario cette réduction serait de $38 \%$ à $31,9 \%$ soit un taux de réduction de $6,1 \%$ (Graphique 3). Quant à la malnutrition aigüe, elle serait réduite de $12,9 \%$ à $9,9 \%$ soit un taux de réduction de $3 \%$ selon les cibles des plans et de $12,9 \%$ à $8,9 \%$ soit un taux de réduction de $4 \%$ selon le scenario (Graphique 4).

Impact des interventions sur la mortalité des enfants de moins de 5 ans selon les plans en vigueur au Mali de 2014 à 2023

Nombre de vies sauvées selon les plans en vigueur de 2014 à 2023 et par intervention

Si les cibles des plans en vigueur étaient atteintes, le nombre de vies sauvées chez les enfants de moins de 5 ans serait de $\mathbf{7 1} \mathbf{5 7 5}$ entre 2014 et 2018 et de 214192 pour la période 2014-2023 (Tableau III).

Les interventions qui contribueraient à sauver plus des vies chez les enfants de moins de 5 étaient le traitement 
du paludisme par une combinaison thérapeutique à base d'artémisinine (34 573 vies sauvées), la possession de moustiquaire imprégnée (27 302 vies sauvées), le vaccin contre $H$ Infleunzae (13 535 vies sauvées), la pris en charge de la septicémie néonatale (12 867 vies sauvées), les pratiques d'allaitement appropriées (11 961 vies sauvées), la prise en charge de travail et l'accouchement (10 956 vies sauvées) et la prise en charge de la malnutrition aigüe sévère (10 424 vies sauvées) (Tableau IV).

En termes de catégorie d'intervention, la prévention et le traitement du paludisme sauveraient 63474 vies soit $30 \%$ du nombre total de vies sauvées chez les enfants de moins de 5 ans. La part des interventions nutritionnelles (pratiques appropriées d'allaitement et d'alimentation de complément, supplémentation en micronutriments et prise en charge de la malnutrition aigüe) serait de 40978 vies soit $19 \%$ des vies sauvées (Graphique 5).

Réduction de la mortalité néonatale, infantile et infanto-juvénile selon les plans en vigueur entre 2014 et 2023

Avec l'atteinte des cibles des plan, la réduction la plus importante s'observerait au niveau de la mortalité infanto-juvénile qui serait réduite de 126,6 à 80 pour 1000 NV soit un taux de réduction d'environ $37 \%$ en 2023. La mortalité infantile serait réduite de 74,7 à 48 pour $1000 \mathrm{NV}$ (soit $36 \%$ de réduction) et la mortalité néonatale de 38,8 à 28 pour 1000 NV (soit $32,5 \%$ de réduction) (Graphique 6).

\section{DISCUSSION}

\section{Limite de l'étude}

Notre étude, comportait un certain nombre de limites qui pouvait être classées en termes de la disponibilité des données et des contraintes de modélisation dans LiST.

Limite en termes de disponibilité des données et choix des indicateurs

Le principal écueil de cette étude est la disponibilité limitée des données de couverture et des cibles des interventions pour les entrées dans le modèle. Certaines interventions spécifiques à la nutrition n'ont pas été modélisées soit par manque de couverture de base, de cible ou parce qu'elles ne sont pas intégrées dans les plans. II s'agit entre autre des interventions d'avant la conception (supplémentation/fortification d'acide folique et en fer), pendant la grossesse (supplémentation en calcium, en micronutriments multiples) et la supplémentation préventive en zinc chez les enfants.

Une autre limite réside dans le choix de la source des données de couverture utilisées pour les niveaux de base par rapport aux projections. L'EDSM 2012 représentait la source de donnée d'enquête la plus récente par rapport à l'année de base (2014). Ainsi, bien que la qualité des données ait été prouvée, l'EDSM V avait la particularité de ne se limiter qu'aux cinq régions du Mali et le district de Bamako. Ces données représentent une période légèrement avant notre base de référence souhaitée 2014.

Limite d'analyse dans LiST

LiST est un outil de modélisation pour guider la planification stratégique et la prise de décision. II ne fournit pas la "vérité absolue". II possède des contraintes inhérentes au modèle, y compris l'incertitude entourant les valeurs par défaut et estimations de l'efficacité, avec certaines, y compris la mortalité et les causes de décès [10]. En outre, LiST est capable de modéliser seulement des interventions avec une voie causale clairement définie. II n'inclut pas les interventions sensibles à la nutrition ainsi que l'iodation du sel et le déparasitage.

Enfin, notre analyse n'a pas abordé les entrées et les processus nécessaires pour atteindre les objectifs de couverture. Les résultats issus de nos projections LiST supposent que les cibles de couverture modélisées seront atteintes.

\section{Principaux Résultats}

Nos résultats montrent que l'atteinte des cibles des plans éviterait plus de 271500 cas de malnutrition chronique entre 2014 - 2018 et plus de 214000 pour la période 2014-2023, mais ne permettrait pas d'atteindre les objectifs de réduction de la sous nutrition et de mortalité. En fait, entre 2014 et 2023, la prévalence de la malnutrition chronique ne serait réduite que de 38 à $33,9 \%$ selon les plans soit un taux de réduction de $11 \%$ et celle de la malnutrition aigüe de 12,9 à 9,9\% soit un taux de réduction de $23 \%$. Ces chiffres sont loin des objectifs fixés dans les plans qui étaient de $15 \%$ pour la malnutrition chronique et de $5 \%$ pour la malnutrition aigüe en 2014. En fait, pour réduire la malnutrition nutrition chronique de 38 à $15 \%$ en 2018 , il faut une réduction de $61 \%$ en 2018 . Ce résultat met en évidence la problématique de la pertinence des cibles et la disponibilité des données. En effet, ces faibles progrès enregistrés pourraient s'expliquer d'une part par le fait que certaines interventions spécifiques avec efficacité prouvée n'ont pas été prises en compte dans le modèle et d'autre part par le fait que les interventions ne concernaient que le secteur de la Santé et du WASH.

Par ailleurs, l'évaluation du bénéfice potentiel maximum des interventions du secteur Santé et WASH à travers le scenario a montré que l'intensification des interventions à une couverture de $100 \%$ permettrait de réduire la malnutrition chronique de $38 \%$ à $31,9 \%$ soit un taux de réduction de $16 \%$. Ce taux est plus élevé que celui des plans (11\%) mais en dessous de celui rapporté par les premières études de Bhutta et coll, selon les quelles, la prévalence du retard de croissance pourrait être réduite 
de $20 \%$ si l'on pouvait étendre la couverture actuelle des dix (10) interventions nutritionnelles prouvées efficaces à une couverture de $90 \%$ de la population actuelle [11]. Cependant il est comparable à ceux rapportés par Zulfiqar Bhutta et coll, qui ont examiné l'impact de l'élargissement de la couverture de grands programmes spécifiques à la nutrition sur le retard de croissance et l'émaciation au Bangladesh, Éthiopie et au Pakistan ainsi que de programmes d'optimisation des intervalles entre les naissances et d'amélioration de l'approvisionnement en eau, de l'assainissement et de l'hygiène[12]. Ils trouvent qu'avec l'élargissement de la couverture des 12 programmes, le modèle évalue les réductions de la prévalence du retard de croissance à $17 \%, 21 \%$ et 18 \% respectivement au Bangladesh, en Éthiopie et au Pakistan de 2013 à 2025 [12].

Ces études montrent que, même si le potentiel des interventions est fonction du contexte, la contribution maximale des interventions du secteur Santé et WASH est rapportée par Butta et coll., avec $20 \%$ de réduction de la prévalence de la malnutrition chronique. Ainsi, s'atteler aux facteurs sous-jacents de la sous nutrition, est fondamental pour couvrir les $80 \%$ restants.

Cette contribution des autres secteurs est rapportée aussi par plusieurs études. Selon Ruel et Alderman, les interventions et programmes contribuant à la nutrition dans le secteur agricole, les filets sociaux, le développement de la petite enfance et l'éducation ont un énorme potentiel pour accroître la portée et l'efficacité des interventions spécifiques à la nutrition [13]. En 2013, la Banque mondiale rapporte que $43 \%$ de la réduction de la dénutrition chez l'enfant est due au niveau d'éducation, $26 \%$ à la disponibilité des aliments, $19 \%$ au contexte sanitaire et $12 \%$ au statut de la femme [14].

En plus de la contribution des autres secteurs sur la sous nutrition, il existe un besoin évident d'introduire des interventions prometteuses reposant sur des preuves pour faire des avancées significatives à court et moyen terme. Notre étude a montré que les pratiques appropriées d'alimentation de complément seraient responsables de plus de $50 \%$ des cas de malnutrition chronique évités chez les enfants de moins de 5 ans. En plus, les interventions nutritionnelles avec $(65 \%)$ et de WASH (31\%) éviteraient $96 \%$ du nombre total de cas de malnutrition chronique. Ces résultats s'expliqueraient par le rôle que jouent l'alimentation de complément et l'hygiène dans la survenue de la malnutrition chronique chez ces enfants. En effet, il est rapporté que l'amélioration du régime alimentaire minimal acceptable est associée à une baisse des taux de l'émaciation et celle de la diversité alimentaire minimale est associée à une baisse des taux de retard de croissance [15]. Selon Victora et coll, la plupart des problèmes entraînant un retard de croissance surviennent entre l'âge de 3 mois et de 18-24 mois, période de vulnérabilité parce que, bien souvent, les enfants reçoivent une alimentation insuffisante et de mauvaise qualité [16]. D'autres études aussi montrent qu'une alimentation d'appoint adaptée, sûre et en quantité suffisante à partir de 6 mois, aboutissait à de meilleurs résultats en termes de santé et de croissance [17].

Toutefois, le risque existe que les aliments d'appoint ne soient pas donnés à l'âge approprié ou selon la fréquence requise ou qu'ils ne soient pas de bonne qualité [18]. Selon Ngure et al, la reconnaissance du rôle fondamental que jouent les mauvaises pratiques dans le secteur de l'eau, l'assainissement et l'hygiène dans l'installation et la perpétuation de la malnutrition est allée croissante ces dix dernières années $[11,19]$.

Concernant la mortalité des enfants, nos résultats ont révélé que, si les cibles des plans étaient atteintes, 214192 vies d'enfants de moins de 5 ans seraient sauvées pour la période 2014-2023. Ce chiffre est en dessous de celui rapporté au cours du cycle 1 de la NEP qui est de 269119 vies sauvées chez les enfants de moins de 5 ans pour la même période [20]. Cette légère différence pourrait s'expliquer par le fait lors de ce cycle 1 , les cibles de malnutrition aigüe $(5 \%)$ et de malnutrition chronique $(15 \%)$ sont rentrées directement dans le modèle alors que dans notre étude se sont les interventions pouvant avoir un impact sur la malnutrition qui étaient modélisées.

Concernant la réduction des taux de mortalité, les gains obtenus en termes de vies sauvées n'ont pas permis de réduire les mortalités de façon conséquente. Notre étude a trouvé que si les cibles des plans étaient atteintes, la réduction la plus importante s'observerait au niveau de la mortalité infanto-juvénile avec un taux de réduction d'environ $37 \%$ (126,6 à 80,2 pour $1000 \mathrm{NV}$ ) mais ne permet pas d'atteindre la cible de $69 \%$ en 2023. Par contre, la mortalité néonatale avec un taux de réduction de $32,5 \%$ (38,8 à 26,2 pour $1000 \mathrm{NV}$ ), la cible de $25 \%$ NV en 2023 est presque atteinte.

Dans notre étude, les interventionnelles nutritionnelles (pratiques appropriées d'allaitement et d'alimentation de complément, supplémentation en micronutriments et prise en charge de la malnutrition aigüe) seraient responsables de $19 \%$ des vies sauvées chez les enfants de moins de 5 ans. L'effet maximal serait observé par la prise en charge de la MAS avec 10424 vies sauvées. Ce chiffre est supérieur à celui de Butta et al, qui trouvent que la mise à échelle des dix (10) principales interventions nutritionnelles identifiées sur une couverture de $90 \%$, pourrait réduire de près de $15 \%$ le nombre de décès des enfants de moins de 5 ans [11]. Selon la même étude, l'effet maximal en termes du nombre de vies sauvées serait observé par la prise en charge de la malnutrition aiguë (435 000) vies sauvées. 
D'où la nécessité de renforcer les programmes de prise en charge de la malnutrition aigüe.

\section{Conclusion}

L'estimation de l'impact potentiel des interventions sur la réduction de la sous nutrition et de la mortalité chez les enfants de moins de 5 ans est nécessaire pour suivre les progrès vers les objectifs de survie de l'enfant et de prioriser les interventions avec plus d'impact.

Notre étude a montré que l'atteinte des cibles des plans en vigueur permettrait d'éviter un nombre important de cas de malnutrition chronique et de sauver plusieurs vies sauvées, mais les gains obtenus seront insuffisants pour réduire de manière considérable la sous nutrition et la mortalité chez les enfants de moins de 5 ans et d'atteindre les objectifs fixés dans les plans.

Les pratiques appropriées d'alimentation de complément permettraient d'éviter plus de la moitié du nombre total de cas de malnutrition chronique chez les enfants de moins de 5 ans.

II ressort aussi de notre analyse que l'intensification des interventions du secteur de la Santé et de WASH est nécessaire, mais même, lorsque leurs taux de couverture atteignaient $100 \%$, n'est pas suffisante pour faire des avancées significatives permettant d'atteindre les cibles des plans. Ainsi, l'étude a mis en évidence l'impérieuse nécessité d'intensifier les interventions du secteur de la Santé et du WASH et d'exploiter le potentiel des interventions sensibles à la nutrition. Seules des actions multisectorielles coordonnées et soutenues prenant en compte les interventions à haut impact permettraient de réduire efficacement les niveaux de la sous nutrition au Mali.

\section{REFERENCES BIBLIOGRAPHIQUES}

1. UNICEF, WHO, World Bank, Levels and trends in child malnutrition. Joint child malnutrition estimates. 2012,

2. Black RE, Victora CG, Walker SP, et le groupe d'étude sur la nutrition maternelle et infantile. Maternal and child under nutrition and overweight in low-income and middle-income countries. Lancet 2013

3. Groupe inter institutions des Nations Unies pour Estimation de la mortalité infantile (UN IGME). Niveaux et tendances de la mortalité infantile ; UNICEF; 2017

4. WHO, UNICEF, UNFPA, World Bank Group, and the United Nations Population Division. Trends in maternal mortality: 1990 to 2015 . Estimates by WHO, UNICEF, UNFPA, World Bank Group and the United Nations Population Division. Geneva: Switzerland; 2015
5. Cellule de Planification et de Statistique du Ministère de la Santé (CPS/MS), Direction Nationale de la Statistique et de I'Informatique (DNSI) et ORC Macro. 2002. Enquête Démographique et de Santé au Mali 2001. Calverton, Maryland, USA : CPS/MS, DNSI et ORC Macro.

6. Ministère de la Santé, Cellule de la Planification et de la Statistique (CPS/SSDSPF), Institut National de la Statistique(INSTAT), Centre d'Etudes et d'Informations Statistiques (INFO-STAT). Enquête Démographique et de Santé du Mali 2012-2013.

7. Groupe inter institutions des Nations Unies pour Estimation de la mortalité infantile (consultation en ligne d'IGME) http://www.childmortality.org/index.php?r=site/gra ph\#ID=MLI_Mali consulté le 8 janvier 2017

8. Ministère de la Santé et de l'Hygiène Publique, Plateforme Nationale d'Evaluation (NEP), Rapport du cycle 1, Mali mai 2015.

9. Walker N, Tam Y, Friberg IK. Overview of the lives saved tool (LiST). BMC. Public Health. 2013;13 Suppl 3:S1. 8 traduit

10. You D, Hug L, Ejdemyr S, Idele P, Hogan D, Mathers $\mathrm{C}$, et al. Global, regional, and national levels and trends in under- 5 mortality between 1990 and 2015, with scenario-based projections to 2030: a systematic analysis by the UN interagency Group for Child Mortality Estimation. Lancet. 2015;386(10010):2275-8

11. Bhutta, Z. A., J. K. Das, A. Rizvi, M. F. Gaffey, N. Walker, S. Horton, P. Webb, et al. 2013a. "Evidence-Based Interventions for Improvement of Maternal and Child Nutrition: What Can Be Done and at What Cost?" The Lancet 382 (9890): 452477.

12. Bhutta, Z. A., J. K. Das, N. Walker, A. Rizvi, H. Campbell, I. Rudan, et R. E. Black. 2013b. "Interventions to Address Deaths from Childhood Pneumonia and Diarrhoea Equitably: What Works and at What Cost?" The Lancet 381 (9875): 14171429.

13. Ruel MT, Alderman $\mathrm{H}$ et le groupe d'étude sur la nutrition maternelle et infantile. Nutrition-sensitive interventions and programmes: how can they help to accelerate progress in improving maternal and child nutrition? Lancet 2013 ; publié en ligne le 6 juin.

14. Banque mondiale. Améliorer au moyen d'Approches Multisectorielles, 2013.

15. Rapport Mondial Nutrition. Mesures et Redevabilité en vue d'accélérer les progrès mondiaux en matière de nutrition 2014. 
16. Victora et coll. Retard de croissance dans les pays en développement. 2010.

17. Bhutta Zulfiqar A., et al., What Works? Interventions for maternal and child undernutrition and survival, The Lancet, vol. 371, no ${ }^{\circ} 9610$, février 2008, p. 417-440.

18. Fonds des Nations Unies pour l'enfance, Guide de programmation, Alimentation du nourrisson et du jeune enfant, UNICEF, New York, juin 2012.

19. Ngure, F. M., B. M. Reid, J. H. Humphrey, M. N. Mbuya, G. Pelto et R. J. Stoltzfus. 2014. "Water,
Sanitation, and Hygiene (WASH), Environmental Enteropathy, Nutrition, and Early Child Development: Making the Links." Annals of the New York Academy of Sciences 1308 (1): 118128.

20. Keita $Y$, Sango H, Roberton T, Vignola E, Traoré M, Munos M et al. Using the Lives Saved Tool to aid country planning in meeting mortality targets: a case study from Mali and for the Mali NEP Working Group.

Liste des tableaux et figures

Tableau I : Niveaux de base et cibles des interventions en 2018 et 2023

\begin{tabular}{|c|c|c|c|c|}
\hline \multirow{2}{*}{ Interventions } & \multirow{2}{*}{$\begin{array}{c}\text { Niveau de base } \\
2014\end{array}$} & \multicolumn{2}{|c|}{ Cibles des plans } & \multirow{2}{*}{$\frac{\text { Scenario }}{2023}$} \\
\hline & & 2018 & 2023 & \\
\hline \multicolumn{5}{|l|}{ Periconceptuelle } \\
\hline Prévalence contraceptive & 10,3 & 15 & 20 & 20 \\
\hline \multicolumn{5}{|l|}{ Grossesse } \\
\hline Soins prénataux & 41 & 65 & 100 & 100 \\
\hline Anatoxine tétanique & 42,3 & 65 & 100 & 100 \\
\hline $\begin{array}{l}\text { TPI - Traitement préventif intermittent pendant la } \\
\text { grossesse }\end{array}$ & 34,6 & 80 & 100 & 100 \\
\hline Supplémentation en fer pendant la grossesse & 67,9 & 80 & 94 & 100 \\
\hline \multicolumn{5}{|l|}{ Naissance } \\
\hline Assistance des naissances par du personnel qualifié* & 58,6 & 67 & 90 & 90 \\
\hline Accouchement dans un établissement de santé & 55 & 65 & 85 & 85 \\
\hline Soins Obstétricaux et Néonataux de Base (SONUB) & 9 & 20 & 30 & 30 \\
\hline \multicolumn{5}{|l|}{ Allaitement } \\
\hline Allaitement exclusif (0-1 mois) & 59 & 68 & 80 & 100 \\
\hline Allaitement exclusif (1-5 mois) & 31 & 50 & 80 & 100 \\
\hline Initiation précoce à l'allaitement (heure qui suit) & 57,8 & 95 & 97 & 100 \\
\hline \multicolumn{5}{|l|}{ Préventif } \\
\hline Soins postnatals & 15,6 & - & 45 & 100 \\
\hline \multicolumn{5}{|l|}{ Alimentation et suppléments } \\
\hline Diversité alimentaire minimale (6 à 23 mois) & 21,6 & 45 & 75 & 100 \\
\hline Supplémentation en vitamine $A$ ( 6 et 59 mois) & 60,8 & 100 & 100 & 100 \\
\hline \multicolumn{5}{|l|}{ Eau, Assainissement et Hygiène } \\
\hline Utilisation d'une source d'eau améliorée & 66,4 & - & 80 & 100 \\
\hline Utilisation d'un raccordement d'eau dans la maison & 8,8 & - & 20 & 100 \\
\hline Utilisation de latrines ou de toilettes (améliorées) & 23,8 & - & 30 & 100 \\
\hline Lavage des mains avec du savon & 17 & & 50 & 100 \\
\hline Evacuation hygiénique des selles des enfants & 59,3 & - & 80 & 100 \\
\hline Possession de MIl par le ménage & 84,37 & - & 100 & 100 \\
\hline \multicolumn{5}{|l|}{ Vaccins } \\
\hline BCG - Une dose & 87 & 95 & 98 & 100 \\
\hline Polio -Trois doses & 81 & 95 & 98 & 100 \\
\hline DTCoq - Trois doses & 74 & 95 & 98 & 100 \\
\hline H. Infleunzae - Trois doses & 74 & 95 & 98 & 100 \\
\hline Hep B - Trois doses & 74 & 95 & 98 & 100 \\
\hline Pneumocoque - Trois doses & 74 & 95 & 98 & 100 \\
\hline Rougeole - Une dose & 72 & 95 & 98 & 100 \\
\hline Rotavirus - Deux doses & 13 & 60 & 60 & 100 \\
\hline
\end{tabular}




\begin{tabular}{lllll}
\hline \multirow{2}{*}{ Interventions } & Niveau de base & Cibles des plans & Scenario \\
\cline { 2 - 4 } & 2014 & 2018 & 2023 & 2023 \\
\hline
\end{tabular}

Curatif

Soins thermiques

Prise en charge de la septicémie chez le nouveau - né

55

55

$S R O$ pour traitement de la diarrhée

Antibiotiques pour le traitement de la diarrhée

Zinc pour le traitement de la diarrhée

Antibiotiques Oraux pour la pneumonie

Vitamine A pour le traitement de la rougeole

CTA- Polythérapie à base d'artémisinine

36,8

15,4

2,1

27,8

60,8

MAS - Traitement de la malnutrition aigüe sévère

15,4

MAS - Traitement de la malnutrition aigüe modérée

22,3

22,3

$\begin{array}{ccc}60 & 85 & 85 \\ 60 & 85 & 85 \\ - & 50 & 100 \\ - & 30 & 100 \\ - & 30 & 100 \\ - & 40 & 100 \\ 100 & 100 & 100 \\ - & 45 & 100 \\ - & 80 & 100 \\ - & 80 & 100\end{array}$

${ }^{*} A$ partir de cet indicateur beaucoup d'autres sont calculés automatiquement par LiST comme soins thermiques, prise en charge de la septicémie.

Tableau II : Nombre de malnutrition chronique évité chez les enfants de moins de 5 ans

\begin{tabular}{lcccc}
\hline \multirow{2}{*}{ Cibles } & \multicolumn{4}{c}{ Cas de malnutrition chroniques évités } \\
\cline { 2 - 5 } & $\mathbf{2 0 1 4 - 2 0 1 8}$ & $\%$ & $\mathbf{2 0 1 4 - 2 0 2 3}$ & $\%$ \\
\hline $0-23$ mois & 66282 & 57 & 320837 & 45 \\
$24-59$ mois & 49584 & 43 & 390365 & 55 \\
$0-59$ mois & 115866 & 100 & 711202 & 100 \\
\hline
\end{tabular}

Tableau III : Nombre de vies sauvées entre 2014 et 2023 selon les plans en vigueur chez les enfants de moins de 5ans

\begin{tabular}{lcc}
\hline \multirow{2}{*}{ Cible } & \multicolumn{2}{c}{ Nombre de Vies Sauvées } \\
\cline { 2 - 3 } & $\mathbf{2 0 1 4} \mathbf{- 2 0 1 8}$ & $\mathbf{2 0 1 4 - 2 0 2 3}$ \\
\hline Nouveau-né (<1 mois) & 6912 & 51769 \\
Enfant de1-59 mois & 64663 & 162423 \\
$0-59$ mois & 71575 & $\mathbf{2 1 4} 192$ \\
\hline
\end{tabular}


Tableau IV : Nombre de vies sauvées chez les enfants de moins de 5 ans par intervention entre 2014 et 2023

\begin{tabular}{lcc}
\hline Interventions & Vies sauvées & $\%$ \\
\hline CTA pour traiter le paludisme & 34573 & 16,2 \\
Possession de MII & 27302 & 12,8 \\
Vaccin H. Influenzae & 13535 & 6,3 \\
Prise en charge de la septicémie néonatale & 12867 & 6,0 \\
Pratiques d'allaitement appropriées & 11961 & 5,6 \\
Prise en charge de travail et l'accouchement & 10956 & 5,1 \\
MAS-Traitement de la malnutrition aiguë sévère & 10424 & 4,9 \\
Vaccin contre le pneumocoque & 9321 & 4,4 \\
Lavage des mains avec du savon & 8223 & 3,8 \\
Supplémentation en vitamine A & 6403 & 3,0 \\
Réanimation néonatale & 6208 & 2,9 \\
Vaccin contre le Rotavirus & 5141 & 2,4 \\
SRO - solution de réhydratation orale & 5117 & 2,4 \\
Alimentation de complément & 4070 & 1,9 \\
Antibiotiques oraux pour la pneumonie & 4048 & 1,9 \\
Pratiques post-natal propre & 3913 & 1,8 \\
Raccordement d'eau dans la maison & 3876 & 1,8 \\
Pratiques d'accouchement propre & 3757 & 1,8 \\
MAM-Traitement de la malnutrition aiguë modérée & 3324 & 1,6 \\
Autres* & 28686 & 13,4 \\
Total & 213705 & 100 \\
\hline
\end{tabular}

*Evaluation et stimulation immédiate ; Vaccin contre la rougeole ; PTME - du VIH ;; Gestion des cas de bébés prématurés ; Vaccin DPT ; Évaluation et stimulation immédiates ; Évacuation hygiénique des selles des enfants ; Anatoxine tétanique ; Zinc pour le traitement de la diarrhée ; Vitamine A pour le traitement de la rougeole ; TPI - pendant la grossesse ; Supplément de micronutriments (fer et autres) ; Amélioration de l'assainissement ; Antibiotiques pour RPM avant terme ; Cotrimoxazole ; Utilisation d'une source d'eau améliorée ; Age maternel et ordre de naissance ; Antibiotiques pour traitement de la dysenterie. 


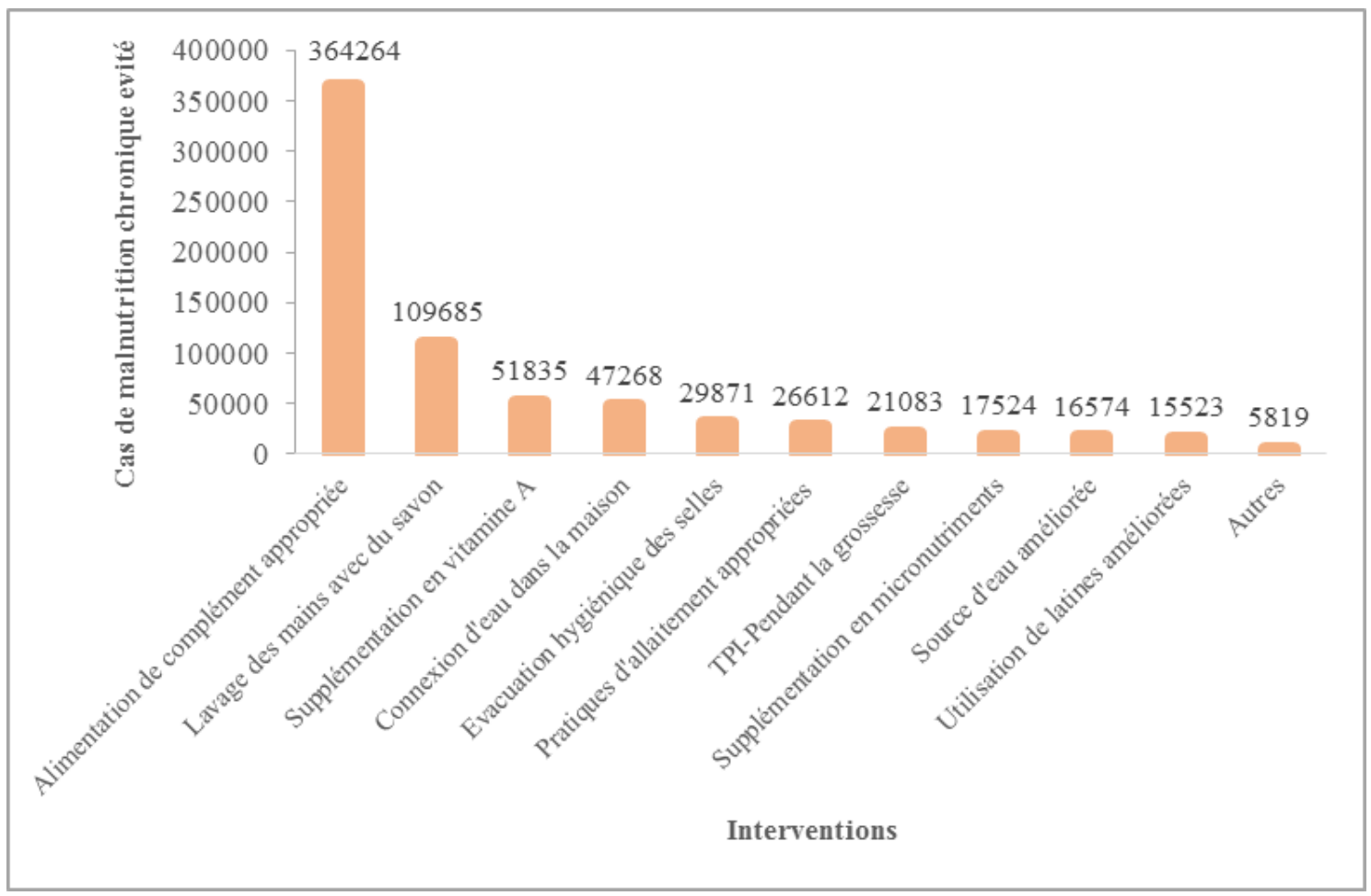

Graphique 1 : Nombre de cas de malnutrition chronique evités par intervention entre 2014 et 2023 chez les enfants de moins 5 ans

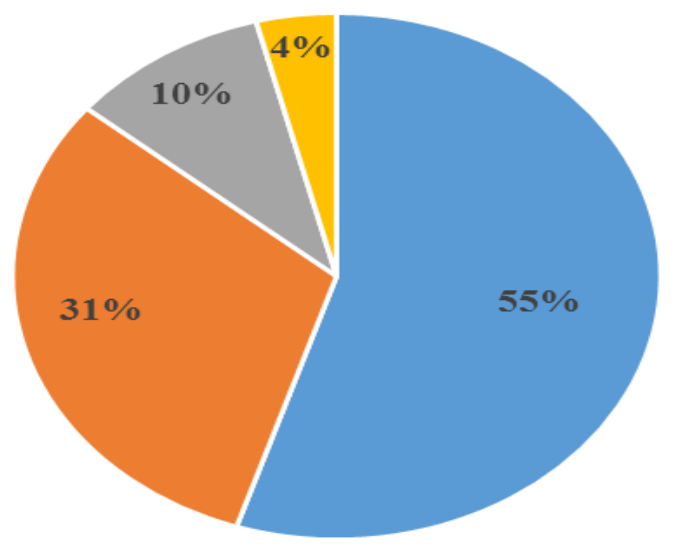

- ANJE

- WASH

- Micronutriments

- Autres

Graphique 2 : Nombre de cas de malnutrition chronique évités chez les enfants de moins de 5 ans par catégorie d'intervention. 


\section{MALI SANTE PUBLIQUE 2020 SOMASAP}

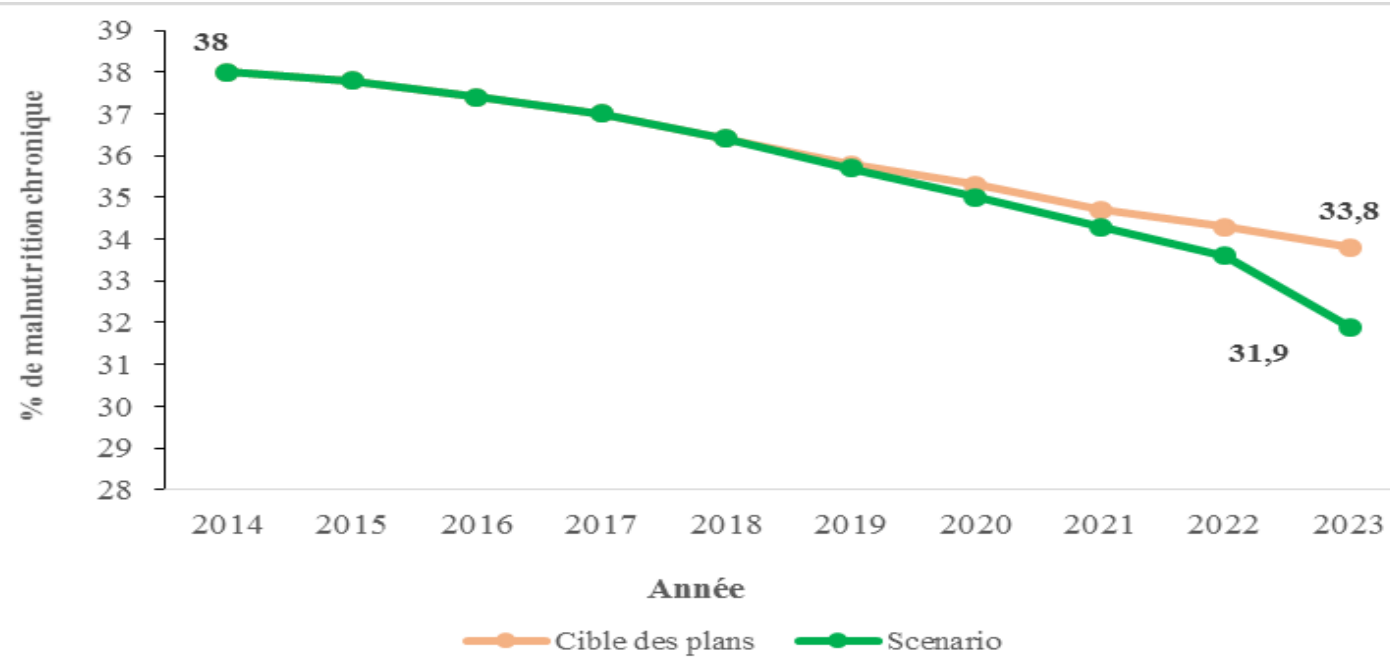

Graphique 3 : Changement de la malnutrition chronique selon les plans en vigueur et le scénario entre 2014 et 2023

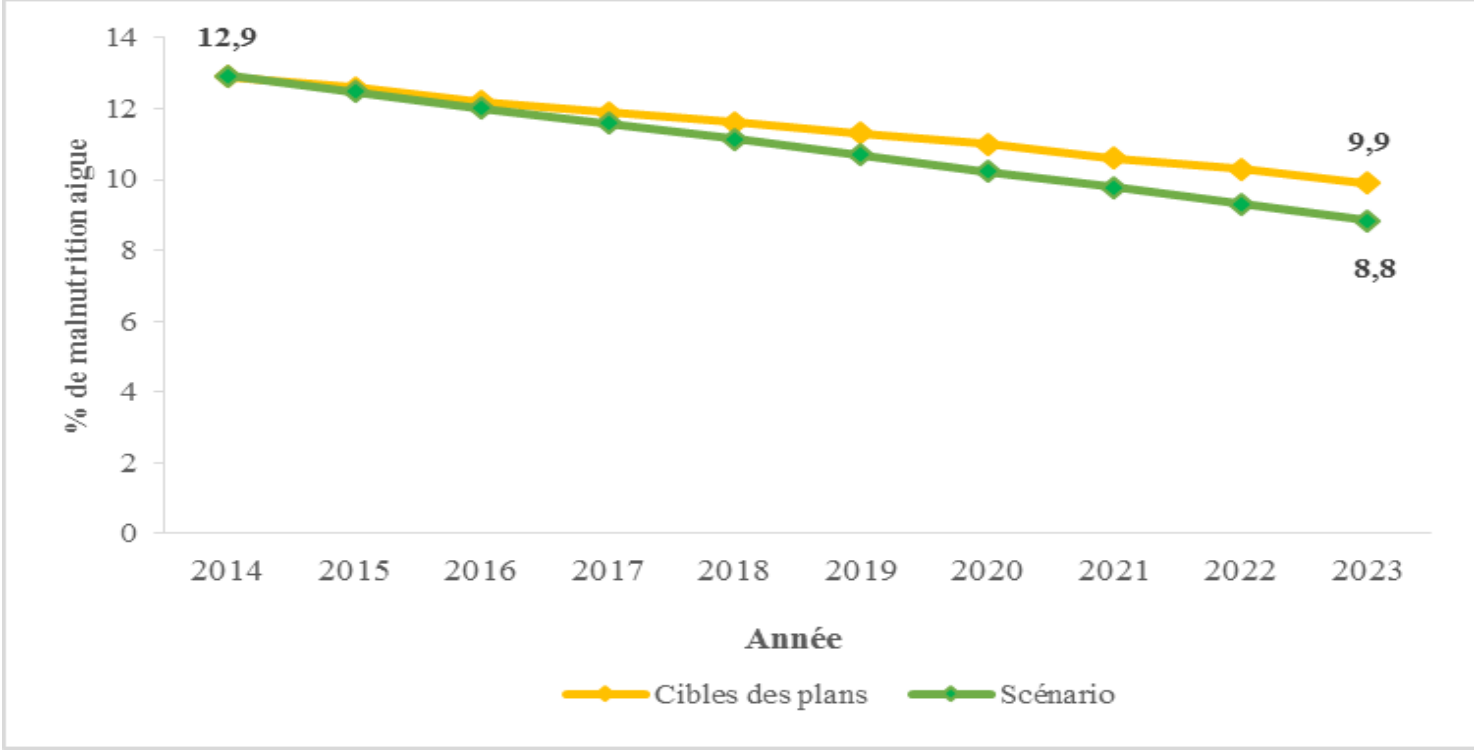

Graphique 4 : Changement de la malnutrition aigüe selon les plans en vigueur et le scénario entre 2014 et 2023 


\section{MALI SANTE PUBLIQUE 2020 SOMASAP}

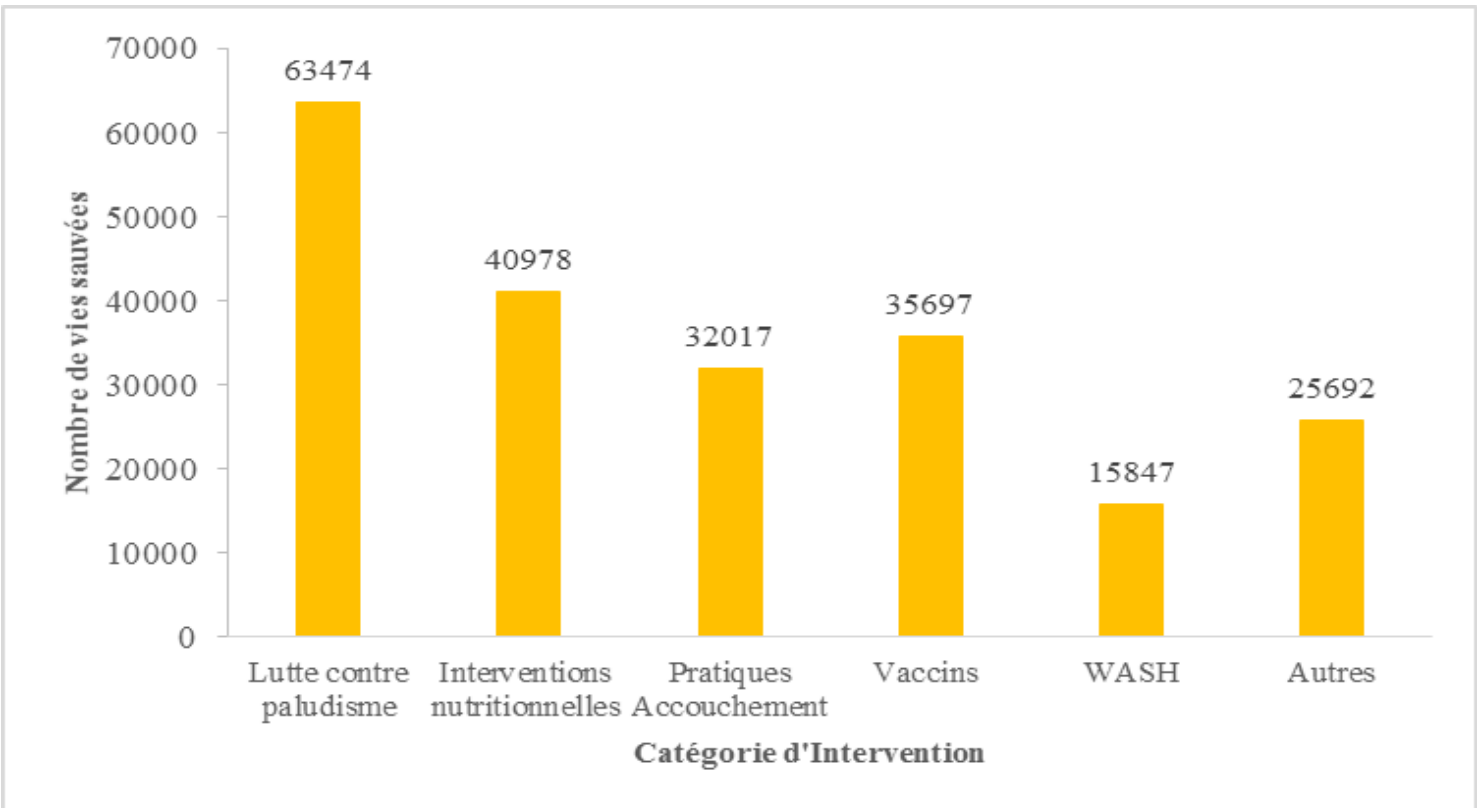

Graphique 5 : Nombre de vies sauvées par catégorie d'interventions chez les enfants de moins de 5 ans entre 2014 et 2023

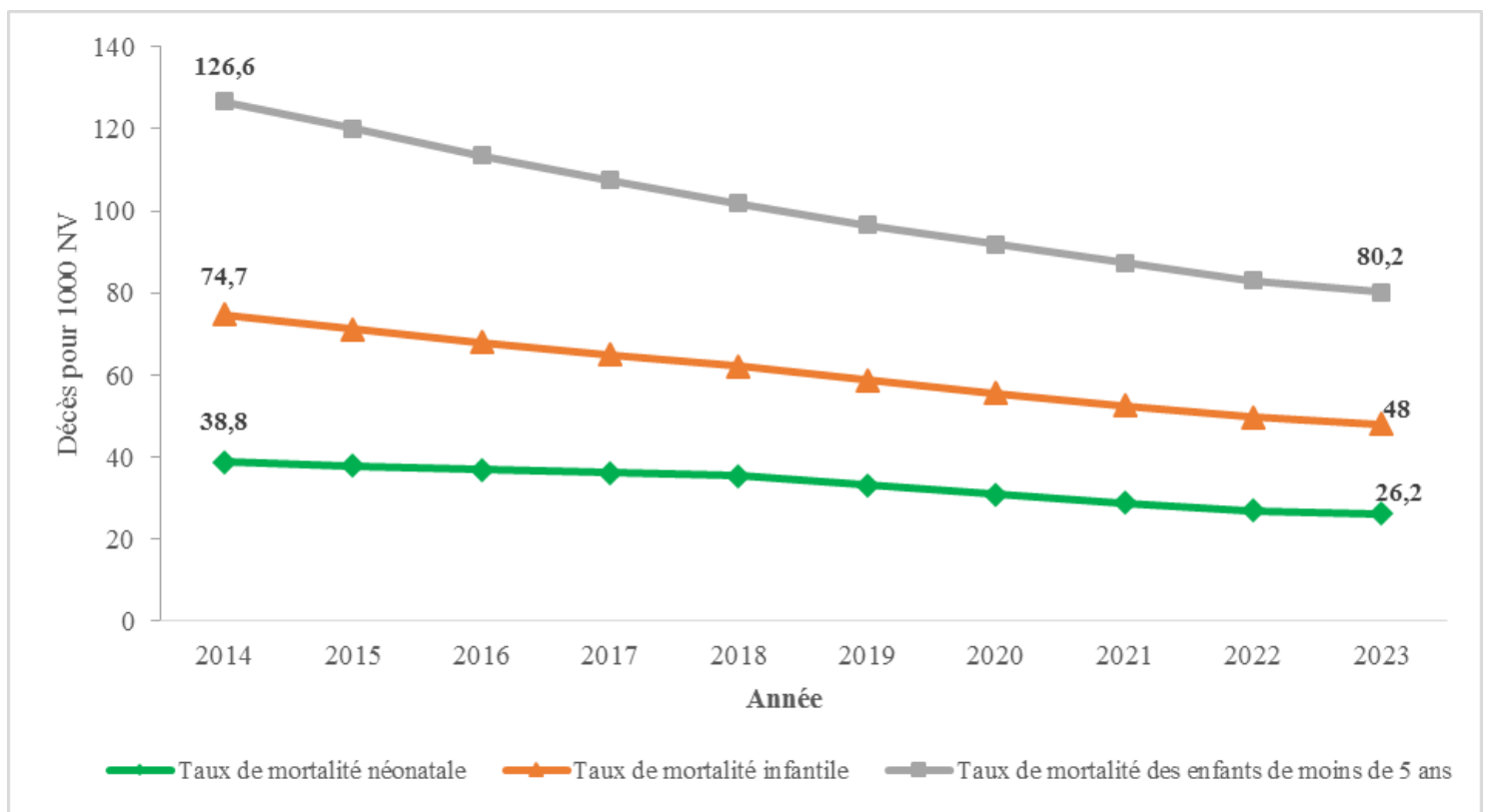

Graphique 6 : Changement de la mortalité néonatale, infantile et infanto-juvénile entre 2014 et $2013 a u$ Mali 